Cinémas

Revue d'études cinématographiques

Journal of Film Studies

\title{
Direct, narration simultanée : frontières de la temporalité
}

\section{François Jost}

Volume 5, numéro 1-2, automne 1994

Le Temps au cinéma

URI : https://id.erudit.org/iderudit/1001006ar

DOI : https://doi.org/10.7202/1001006ar

Aller au sommaire du numéro

Éditeur(s)

Cinémas

ISSN

1181-6945 (imprimé)

1705-6500 (numérique)

Découvrir la revue

Citer cet article

Jost, F. (1994). Direct, narration simultanée : frontières de la temporalité.

Cinémas, 5(1-2), 81-90. https://doi.org/10.7202/1001006ar

\section{Résumé de l'article}

L'auteur avance l'idée que le film appartient à la classe des empreintes par connexion physique produites dans le passé, le " direct ", à celle des empreintes par connexion physique produites dans le présent. Dans cette fissure sémiotique d'ordre temporel et non plus seulement aspectuel s'immisce une différence d'auteurisation. Le temps filmique va toujours vers son destin parce qu'il obéit à un ordre supérieur, à une intentionnalité humaine; le temps du direct, lui, n’a qu'un avenir et il « adhère " si fondamentalement à la réalité qu'il le crédite toujours de lui faire vivre des événements qui n'ont pas encore été écrits, quand bien même ils seraient prévisibles. L'auteur tente ici de démontrer que, face aux images de reportage, nous réagissons de la même manière que face au monde. 


\section{Direct, narration simultanée : frontières de la temporalité}

\section{François Jost}

\section{RÉSUMÉ}

Lauteur avance l'idée que le film appartient à la classe des empreintes par connexion physique produites dans le passé, le "direct", à celle des empreintes par connexion physique produites dans le présent. Dans cette fissure sémiotique d'ordre temporel et non plus seulement aspectuel s'immisce une différence d'auteurisation. Le temps filmique va toujours vers son destin parce qu'il obéit à un ordre supérieur, à une intentionnalité humaine; le temps du direct, lui, n'a qu'un avenir et il "adhère" si fondamentalement à la réalité qu'il le crédite toujours de lui faire vivre des événements qui n'ont pas encore été écrits, quand bien même ils seraient prévisibles. Lauteur tente ici de démontrer que, face aux images de reportage, nous réagissons de la même manière que face au monde.

\section{ABSTRACT}

The author argues that film belongs to the class of traces made by physical connection in the past, and live broadcast to that of traces made by physical connection in the present. This semiotic fissure of a temporal order, not just an aspectual one, is intermixed with a difference in authoring. Filmic time always proceeds towards its destiny, since it obeys a higher order, a human intentionality, while "live" time just has a future and it "adheres" so closely to reality that it always credits the future for keeping it alive with events that have not yet been recorded, even though they may be foreseeable. The author is trying to show here that when confronted with news images, we react the same way that we react to the world. 
Y a-t-il un temps de l'image-mouvement en général, par opposition à une temporalité de l'image fixe? Ou, pour dire les choses autrement, toutes les images animées représentent-elles le temps de la même façon? Je vois au moins deux façons de répondre par l'affirmative à de telles questions.

La première, s'appuyant sur une analyse quasi ontologique du langage visuel, consiste à trouver dans le mouvement une capacité représentative différente de celle de la fixité; on soutient alors que l'image-mouvement a bouleversé la question liée à la représentation de l'instant, d'une part à cause de sa capacité à restituer le temps de l'événement de façon exhaustive, d'autre part du fait de l'obligation résultant de son dispositif de nier l'instantané et l'instant représentatif. L'image-mouvement serait un dispositif à reproduire le quelconque ${ }^{\prime}$.

La seconde, qui émane de la narratologie, tire des arguments, par exemple, du fait que, quel que soit le médium mis en jeu, les combinaisons du récit verbal et de l'image peuvent s'appréhender au moyen des mêmes instruments conceptuels : ainsi, le concept de narration simultanée s'appliquerait aussi bien à un récit fictif comme $E$ la nave va (Fellini, 1983), où un chroniqueur décrit ce qu'il voit, qu'au récit factuel du reporter commentant des événements en direct. En somme, les catégories temporelles forgées par la sémiologie ou la narratologie seraient valables pour n'importe quel média fondé sur l'image animée.

Ces deux positions, malgré leurs différences, ont un point commun : elles mettent le temps dans la chose elle-même, celuici relevant, en fin de compte, de la "part de l'image ${ }^{2}$ ». Si tournant le dos à une telle conception, on pense plutôt le temps comme un cadre cognitif nécessaire au spectateur pour comprendre ce qu'il voit et ce qu'il entend, la thèse de l'unicité du temps des images animées devient difficilement défendable. Le temps se diffracte et prend des formes diverses selon qu'il s'agit d'images cinématographiques, vidéographiques ou télévisuelles.

Voyons d'abord l'argument, tiré d'une réflexion sur le dispositif, selon lequel toute image-mouvement bouleverse la représentation de l'instant, par son incapacité à fixer cet instant prégnant dont parlait Lessing. Pour évaluer une telle conclusion, il convient d'abord de remarquer que, pour le critique allemand, la 
théorie de l'instant prégnant n'est nullement un constat d'ordre sémiologique, comme on le laisse entendre trop souvent. On ne saurait négliger non plus que, même si la pensée de la peinture occupe une place primordiale dans le Laocoon, son point de départ est une sculpture, comme l'indique le titre de l'ouvrage, c'est-à-dire une œuvre en trois dimensions. C'est donc la fixité qui retient l'attention de Lessing, et non l'image. Si, pour lui, l'arrêt du temps fait question, c'est moins pour des raisons de signification que pour des raisons d'expressivité; le souci du Laocoon n'est pas de déterminer l'instant qui résumerait "la signification d'un événement réel " comme le soutient Aumont (p. 180), mais de cerner quelle est la représentation de l'instant qui crée chez le spectateur l'intensité émotionnelle maximale. Lalternative est, en fait, la suivante: l'artiste doit-il garder une certaine mesure dans la représentation de l'événement ou choisir "le suprême degré d'intensité " (Lessing, p. 67) ? La réponse est claire: "Présenter aux yeux le degré extrême, c'est lier les ailes à l'imagination" (p. 68). C'est au spectateur d'imaginer, de concevoir les instants extrêmes; la représentation des événements est moins dans les choses que dans sa tête.

L'édiction de cette loi repose sur l'argumentation suivante: l'art étant une durée immuable, il ne doit pas "exprimer ce qui ne se conçoit que comme transitoire", il doit représenter ce que la théorie du récit appelle un "état". Pourquoi? Parce que l'œuvre étant par nature faite pour être revue, l'impression provoquée par une représentation de l'instant paroxystique irait s'amenuisant. "La Mettrie, qui s'est fait peindre et graver en Démocrite, ne rit que la première fois qu'on le voit. Regardez-le davantage: le philosophe devient un niais et son rire un ricanement " (Lessing, p. 69).

Contrairement au temps de la peinture qui est par nature iconique, c'est-à-dire re-présentation d'un instant unique construit et choisi, le temps de l'image photographique, animée ou non, est indiciel même si le montage peut former une temporalité iconique. Ce constat sémiotique signifie-t-il pour autant que l'image-mouvement soit " une négation de la technique de l'instantané, de l'instant représentatif" (Aumont, p. 181), condamnée à reproduire des instants quelconques? 
Il n'est que de comparer le temps mécanique de l'enregistrement et le temps filmique pour se convaincre qu'on ne saurait déduire d'un fait de langage des conséquences esthétiques. Bien sûr, certaines utilisations de la caméra collent au plus près de la banalité du quotidien, enregistrant comme un flot continu une succession quelconque d'entrées et de sorties de champ de quidams dans les couloirs des métros ou dans les agences bancaires.

Ce dispositif machinique n'a cependant rien à voir avec le cinéma ou la télévision. Même un film limite comme Empire (Warhol, 1964) ne s'interprète pas comme une bande enregistrée par une caméra de surveillance parce que, à partir du moment où je vais le voir, je l'interprète comme une durée intentionnellement construite et non comme une simple reduplication du monde (de la même façon, le fait qu'une boîte de Coca Cola soit exposée dans un musée transfigure le banal, comme dirait Danto ${ }^{3}$ ). Regardons les "vues" des opérateurs Lumière: malgré la banalité apparente du quotidien qu'elles captent, en associant telles images à telle activité ou profession, en choisissant tel moment plutôt que tel autre, elles renvoient implicitement la durée du film à une temporalité qui les excède et qu'elles concentrent néanmoins. Que la durée d'un plan soit isochrone à l'événement qu'il représente (c'est une des caractéristiques de la fameuse "monstration" d'André Gaudreault) ne suffit donc pas à nous sortir de la problématique de l'instant représentatif puisque le spectateur n'interprète pas la durée de la même manière, selon qu'il la pense comme intentionnellement construite ou comme extraite au déroulement du monde.

Dans cette optique, on doit bien admettre qu'évacuer la problématique de "l'instant unique" pour le seul fait que l'image animée a la faculté langagière de reproduire tous les instants, c'est-à-dire une durée quelconque, c'est faire fi à la fois de la portée esthétique de la théorie de Lessing qui, comme on l'a vu, ressortit à la question de l'expressivité et de l'anthropomorphisme régulateur qui transforme, chez le spectateur, le dispositif machinique en une intentionnalité humaine. Quoi qu'il en soit de la durée d'un plan, il est toujours loisible de le considérer comme un punctum temporis, une photographie au temps de pose distendu (n'appelait-on pas le cinématographe "photographie ani- 
mée" ?), à partir du moment où je l'intègre au discours d'un sujet qui sélectionne et découpe la temporalité du monde. Dès lors, l'expressivité de ce punctum temporis, eu égard à l'émotion que l'on veut susciter chez le spectateur, est tout aussi problématique pour les images animées que pour les images de la fixité.

Faire un scénario, c'est toujours donner raison à Lessing, puisque c'est admettre que la représentation d'une durée $x$ est à même de recréer à la fois l'environnement cognitif et émotionnel des personnages, dont on peut supposer parfois qu'il a pour cadre une temporalité plus vaste. Peu importe la longueur de ce temps: il peut se rapprocher de zéro, de la fixité; il suffit souvent d'un plan de regard, pour que le spectateur infere la tristesse ou la joie qui envahit un personnage (que l'on pense au visage de Giulette Massina dans La Strada [Fellini, 1954] se retournant vers le monde qu'elle quitte). Le problème qui se pose au metteur en scène est bien celui du Laocoon: quel procédé est-il le plus apte à faire partager au spectateur la douleur d'un personnage? Un cri ? Des larmes? Une réplique? Un geste? Dans ces réponses, qui n'engagent pas forcément le mouvement, se lit une diversité d'esthétiques: du cinéma hollywoodien qui se fait comprendre par le spectateur du fond de la salle, grâce au reaction shot, à un cinéma de la suggestion.

Le fait qu'un réalisateur décide de re-présenter l'événement dans sa durée réelle change-t-il quelque chose à l'hypothèse que j'avance ici? Qu'un film comme Jeanne Dielman (Akerman, 1976) me montre une femme qui épluche des pommes de terre aussi longtemps qu'il est nécessaire pour accomplir cette tâche dans la vie a-t-il pour effet de transformer cette durée en instant quelconque? On serait tenté de répondre par l'affirmative tant ce moment est anodin, sans portée dramatique évidente. Au deuxième examen, on doit cependant admettre qu'étendre le temps à la durée isochrone du geste quotidien est aussi efficace du point de vue de l'expressivité que le plan de Gesolamina regardant derrière elle. Gageons que l'ennui de l'un et la tristesse de l'autre résisteront à l'épreuve du temps: à chaque projection, nous les retrouverons intacts.

Pour montrer qu'il n'est pas juste de réduire l'expressivité temporelle du film aux propriétés intrinsèques de l'image- 
mouvement, j'ai noté que le jugement porté sur la qualité de l'instant représenté était fonction de l'anthropomorphisation opérée ou non par le spectateur, opération consistant comme je l'ai dit, à renvoyer des images à un Je-origine ou, à l'inverse, au seul dispositif machinique ${ }^{4}$. J'irai à présent plus loin dans cette voie pour soutenir l'idée qu'il n'est pas possible d'appliquer les résultats d'une analyse concernant des combinaisons narratologiques des récits verbal et visuel au cinéma à tous les domaines de l'audiovisuel. Un bon champ d'observation est offert par la narration simultanée. Regardons Orlando dans $E$ la nave va, qui commente ce qu'il voit : "Le grand-duc est suivi de sa sœur Lerimia, la princesse aveugle qui a perdu la vue tout enfant. Pourtant, vous avez remarqué, elle marche à côté du premier ministre avec une incroyable assurance..." Ne ressemble-t-il pas comme un frère à Léon Zitrone, ce journaliste de télévision qui décrit ainsi les obsèques du roi Baudouin : "Retour sur les majestés... Juan Carlos, Sophie, Béatrix [...] $x$, qui a connu son mari dans des circonstances extraordinaires. Elle était employée..."

Du point de vue iconique, ces images se valent: qu'il s'agisse du film ou du reportage, les événements nous sont montrés en train de s'accomplir. Contrairement à ce qu'on dit souvent, l'image animée n'est pas plus au présent que l'image fixe, elle donne seulement à voir le procès sous l'angle de son effectuation. En ce sens, rien ne différencie un reportage en direct de ce même reportage vu quelques heures plus tard avec l'inscription direct encore incrustée. Rien si ce n'est le savoir temporel du spectateur concernant l'image animée en tant qu'indice "photonique». De ce point de vue, film et direct ne sauraient être identifiés l'un à l'autre: le premier appartient à la classe des empreintes par connexion physique produites dans le passé, le second à celle des empreintes par connexion physique produites dans le présent.

Dans cette fissure sémiotique d'ordre temporel, et non plus seulement aspectuel, s'immisce une différence d'auteurisation (Jost, p. 66). La compréhension du temps cinématographique repose tout autant sur le fait que, grâce à la "monstration" et à la narration, le film signifie temporellement que sur le savoir qu'on veut me transmettre au moyen du temps cinématographique. 
Et le direct? Veut-on me dire quelque chose par son entremise? Qui en est l'auteur? La réponse est beaucoup moins assurée. Certes, je n'oublie pas que les images sont manipulées par un réalisateur qui joue le rôle du locuteur de la langue; mais cette présence langagière ne va pas jusqu'à annuler le fait que la réalité passe à travers son discours, même si c'est de façon déformée. La réalité y est bien celle de l'énonciateur, au sens que Ducrot donne à ce mot: le réalisateur adopte son point de vue,

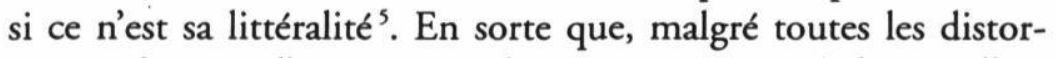
sions qu'impose l'intervention humaine au temps (ralentis, ellipses, discontinuités, etc.), je le construis à l'image d'un temps référentiel que j'imagine aussi intègre que le mien.

De l'auteurisation du film de fiction, j'infere que l'image est toujours au-delà de la simple opération de renvoi : montrer quelqu'un qui épluche des pommes de terre, ce n'est pas nous communiquer la durée de l'épluchage, c'est nous faire accéder à une dimension d'emblée symbolique, qui serait, en l'occurrence, la monotonie du quotidien. Le direct, en revanche, ne nous renvoie qu'au réel et il n'a donc pas plus de sens que celui que nous attribuons au monde.

Cette opposition entre une temporalité renvoyée à une intentionnalité auctoriale et une temporalité subie parce que produite par la suite du monde plus que par une subjectivité qui l'aurait construite de toutes pièces, modifie profondément ma croyance au temps de l'image: tout en n'ignorant pas que celui qui retransmet l'événement le construit aussi bien, je sais que cet événement existerait sans cette retransmission. Le temps filmique va toujours vers son destin, parce qu'il obéit à un ordre supérieur, une intentionnalité humaine; le temps du direct, lui, n'a qu'un avenir et il "adhère " si fondamentalement à la réalité que je le crédite toujours de me faire vivre des événements qui n'ont pas encore été écrits, quand bien même ils seraient prévisibles.

Le direct ne nous ouvre pas pour autant sur l'inconnu et il serait illusoire de confondre ici l'activité narrative d'écriture et l'activité narrative du spectateur qui ne cesse d'anticiper sur ce qu'il voit par une série d'hypothèses. Face aux images du reportage, en effet, nous réagissons de la même manière que face au monde. Ainsi, Léon Zitrone décrit le plan du cercueil de 
Baudouin: "Est-ce que la télévision va nous donner l'instant précis de la levée du corps ou bien, par pudeur, elle va plonger dans la foule?"

Ce que cherche le commentateur, c'est bien à présent cette image qui fixerait le temps, cet instant unique qui en épuiserait le sens. Il s'agit bien, cette fois, de cerner l'instant décisif apte à représenter la signification d'une durée vécue comme quelconque parce que sans ancrage intentionnel, d'extraire du continuum celle qui deviendra "l'image du jour". En somme, c'est l'absence d'intentionnalité qui rend l'instant du direct quelconque et non la nature ou le contenu du temps représenté, comme on peut le croire. En d'autres termes, l'ancrage énonciatif guide notre réception du temps, c'est lui qui donne vie au "temps mort ", si j'ose dire, des obsèques.

Comment opère cette réanimation de l'image animée? Par l'invention d'un récit, c'est-à-dire par la création à la fois d'actions dans ce qui n'est qu'empreinte du réel et d'un temps chronique: "La levée du corps, insiste Léon Zitrone, est prévue

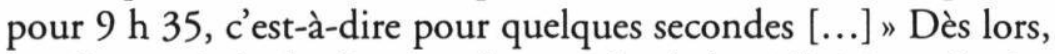
en glissant de la duplication du monde, de la réalité énonciatrice à l'ancrage dans un Je-origine historique, comme dirait Käte Hamburger, le direct devient la vérification d'un temps que nous avons déjà vécu mentalement. C'est là le point saillant qui l'oppose à la temporalité du film, car condensation du temps en un instant décisif ne signifie pas arrêt du temps. Pour cette raison, je distinguerai l'instant décisif de l'instant unique de Lessing: alors que celui-ci immobilise une durée dont l'éternel retour aurait toujours la même charge émotionnelle, celui-là déclenche le temps de la mémoire. Pourquoi isoler l'instant précis de la levée du corps est-il si important? Parce que cet instant est une "scène", qui appartient à ce que Robert C. Schank appelle un MOP (Memory Organization Packet). Avec cette image, nous pourrions nous raconter à nous-mêmes toutes les obsèques, les imaginer: il suffirait de l'intégrer à cette structure mémorielle plus vaste $\mathrm{M}$-Obsèque. L'évoquer verbalement suffit à la faire exister et à nous fournir un fil invisible qui relie tous les instants quelconques qui nous sont donnés à voir. Le spectateur arrête le temps pour se l'approprier et, finalement, pour le 
relancer ou, mieux, pour le vivre. Ce faisant, il élabore une organisation narrative de ce qui est d'abord une empreinte de la réalité. En somme, le je du journaliste de la narration simultanée pense le visible comme un monde organisé, comme un monde diégétique, où chaque séquence apporterait son concours à la connaissance d'un monde unitaire, où chaque séquence serait pertinente ${ }^{6}$. C'est ce comme qui transforme le reportage en fiction. Ce travail de la mémoire existe aussi pour la fiction, mais il est, en quelque sorte, canalisé à la fois par la restriction de l'histoire à des instants représentatifs et par les hypothèses que l'auteur peut faire sur les inférences spectatorielles.

Le narrateur-chroniqueur d' $E$ la nave va mime à sa manière une telle configuration, mais sans jamais l'atteindre. Ce n'est pas le fait qu'il apparaît dans l'image et que, en conséquence, il appartienne au discours d'un narrateur implicite ou d'un méganarrateur qui l'en empêche: après tout, le journaliste de télévision peut aussi apparaître à l'écran et dépendre d'un niveau d'énonciation supérieur où s'organise l'image. C'est plutôt que l'énoncé du chroniqueur ne peut coïncider avec la temporalité de l'empreinte: en donnant l'impression de découvrir du vu, dont nous savons qu'il est prise de vue, le je-narrateur perd donc cette ambiguïté qui, selon K. Hamburger, rend parfois difficile d' "établir avec certitude la frontière qui sépare le récit à la première personne de l'autobiographie authentique" (p. 295). Car, contrairement à ce qui se passe du côté littéraire, où le temps verbal désigne le temps dans lequel vit cette première personne, au cinéma, le temps de la narration verbale est altéré par celui de l'empreinte. Le présent de l'énonciation narrative ne peut que contredire le passé du temps cinématographique et son renvoi à un Je-origine disparu.

Direct, narration simultanée : a priori, ces deux phénomènes semblaient entretenir bien des points communs d'un point de vue narratologique. Ce qui les rapproche n'existe qu'aussi longtemps qu'on reste dans un cadre d'interprétation immanentiste peu soucieux des phénomènes d'interprétation. Il faut s'y résoudre: les frontières de la temporalité ne passent pas par le film, mais par le spectateur.

Université de Paris III (Sorbonne Nouvelle)

Direct, narration simultanée: frontières de la temporalité 


\section{NOTES}

1 C'est la position de Jacques Aumont dans L'Image (Paris : Nathan, 1990).

2 Titre du chapitre 4 de l'ouvrage de J. Aumont, L'Image.

3 Voir La Transfiguration du banal (Paris: Seuil, 1989).

4 Sur l'anthropomorphisme, voir Jost, Un monde à notre image (Paris: Méridiens / Klincksieck, 1992).

5 Les énonciateurs sont "ces êtres qui sont censés s'exprimer à travers l'énonciation, sans que pour autant on leur attribue des mots précis" (Le Dire et le Dit, Paris: Minuit, 1984, p. 204). Dans Un monde à notre image, j'utilise ce concept pour définir le point de vue idéologique construit par l'image.

6 Si l'on admet que la pertinence est ce qui vise à améliorer la connaissance de notre environnement... Voir Sperber et Wilson, La Pertinence (Paris: Minuit, 1989).

\section{OUVRAGES Cités}

Aumont, Jacques. L'Image. Paris: Nathan, 1990.

Ducrot, Oswald. Le Dire et le Dit. Paris: Minuit, 1984.

Hamburger, Käte. Logique des genres littéraires. Paris: Seuil, 1986.

Jost, François. Un monde à notre image. Énonciation, cinéma, télévision. Paris: Méridiens/Klincksieck, 1992.

Lessing, G.E. Laocoon. Paris : Hermann, 1964. 